\title{
Device Manufacture Date
}

National Cancer Institute

\section{Source}

National Cancer Institute. Device Manufacture Date. NCI Thesaurus. Code C93804.

The date (and time) on which the medical device is made. 